\title{
VLA observations of water maser emission associated with SVS 13 and other sources in NGC 1333
}

\author{
L. F. Rodríguez ${ }^{1}$, G. Anglada ${ }^{2,3}$, J. M. Torrelles ${ }^{4}$, J. E. Mendoza-Torres ${ }^{5}$, \\ A. D. Haschick ${ }^{6}$, and P. T. P. Ho ${ }^{3}$
}

1 Instituto de Astronomía, UNAM, Campus Morelia, Apdo. Postal 3-72, Morelia, Michoacán 58089, México

2 Instituto de Astrofísica de Andalucía, CSIC, Camino Bajo de Huétor 24, 18008 Granada, Spain e-mail: guillem@iaa.es

3 Harvard-Smithsonian Center for Astrophysics, 60 Garden St., Cambridge, MA 02138, USA e-mail: ganglada, ho@cfa.harvard.edu

4 Institut d'Estudis Espacials de Catalunya (IEEC/CSIC) and Instituto de Ciencias del Espacio (CSIC), Edifici Nexus, c/ Gran Capità 2-4, 08034 Barcelona, Spain e-mail: torrelles@ieec.fcr.es

5 Instituto Nacional de Astrofísica, Optica y Electrónica, Luis Enrique Erro No. 1, Apdo. Postal 51 y 216 , 72840 Tonantzintla, Puebla, México

e-mail: mend@inaoep.mx

${ }^{6}$ MIT Haystack Observatory, Westford, MA 01886, USA

Received 24 October 2001 / Accepted 16 April 2002

\begin{abstract}
The young stellar object SVS 13 is believed to power the chain formed by the HH 7-11 objects. Recent observations have revealed that in the radio continuum $(3.6-\mathrm{cm})$ the source is a binary with components separated by about $0 . " 3$ in the east-west direction. The members of the binary are named VLA 4A (western component) and VLA 4B (eastern component). In an attempt to ascertain which of the two components of the binary is the most likely candidate to drive the outflow, we obtained accurate positions for the $\mathrm{H}_{2} \mathrm{O}$ maser spots toward this source, also known as $\mathrm{H}_{2} \mathrm{O}(\mathrm{A})$, derived from Very Large Array observations in its highest angular resolution A configuration. We detected a total of eleven spots over four observational sessions (made in the years 1983, 1985, 1988, and 1989). The spots are segregated in two velocity groups: a velocity group with $v_{\text {LSR }}$ similar to that of the ambient cloud $\left(v_{\mathrm{LSR}} \simeq 8 \mathrm{~km} \mathrm{~s}^{-1}\right)$, for which we detected five features (with $v_{\mathrm{LSR}}$ in the range of 6.5 to $12.2 \mathrm{~km} \mathrm{~s}^{-1}$ ) and a blueshifted velocity group (six features with $v_{\text {LSR }}$ in the range of -25.4 to $-5.6 \mathrm{~km} \mathrm{~s}^{-1}$ ). Remarkably, all the spots in the first velocity group are associated with VLA 4A, while all the spots in the blueshifted velocity group are associated with VLA 4B. We discuss the possible implications of this observation. We also present accurate positions of the $\mathrm{H}_{2} \mathrm{O}$ maser $\mathrm{HH} 7(\mathrm{~B})$ and of the masers associated with IRAS4B (VLA 28) and IRAS7 (VLA 27, the exciting source of $\mathrm{HH}$ 6).
\end{abstract}

Key words. ISM: Herbig-Haro objects - ISM: jets and outflows - masers - stars: pre-main sequence

\section{Introduction}

NGC 1333 is a reflection nebula associated with a region of recent, very active star formation in the Perseus molecular cloud. A cluster of about 150 low- to intermediate-mass YSOs have been identified in near-infrared images (Aspin et al. 1994). The region also contains about 20 groups of Herbig-Haro (HH) objects, some with highly collimated jets (Bally et al. 1996). Rodríguez et al. (1999) found a total of 44 sources at centimeter wavelengths, most of which are associated or believed to be associated with young objects in the region.

Send offprint requests to: L. F. Rodríguez,

e-mail: luisfr@astrosmo.unam.mx
The best studied HH group in this region is HH 7-11, a classical system of bright $\mathrm{HH}$ objects, first reported by Herbig (1974) and by Strom et al. (1974). Strom et al. (1976) discovered a $2.2 \mu \mathrm{m}$ source, SVS 13, roughly aligned with the chain of $\mathrm{HH}$ objects, that was proposed as their exciting source. Rodríguez et al. (1997), through a high angular resolution VLA map of the region detected SVS 13 as a radio cm source (VLA 4), but also detected a new object, VLA 3 , located $6^{\prime \prime}$ to the SW, and argued that this new object was a better candidate to drive the outflow. Bachiller et al. (2000) find extremely high velocity molecular gas in association with SVS 13 and high velocity gas in the vicinity of both SVS 13 and VLA 3, favoring the former object as the exciting source of the 
HH 7-11 outflow. Deep radio maps with subarcsecond angular resolution obtained by Anglada et al. (2000) revealed that the radio source associated with SVS 13 (VLA 4) is actually a binary (VLA 4A and 4B), separated by 0 "' 3 , corresponding to a projected separation of $65 \mathrm{AU}$ for a distance of 220 pc (Černis 1990).

The source SVS 13 presents a number of peculiar properties. It was detected initially as a near-IR source (Strom et al. 1976). Later, Goodrich (1986) detected a faint visible counterpart. The source exhibited a large increase of its brightness in the optical and near-IR in 1988-1990 (Eislöffel et al. 1991). Since then, the flux remained almost steady (Aspin \& Sandell 1994). In addition, despite being optically visible, SVS13 is a strong mm source (Grossman et al. 1987; Chini et al. 1997; Bachiller et al. 1998; Looney et al. 2000). Anglada et al. (2000) have argued that the two components of this binary system could be in different evolutionary stages and/or suffer from different extinction, with the more extincted object (proposed to be VLA 4B by Anglada et al.) being associated with the previously reported mm source, while the other component (VLA 4A) would correspond to the visible star SVS 13.

In order to further investigate SVS13, we have analyzed archive Very Large Array (VLA) observations of the $\mathrm{H}_{2} \mathrm{O}$ maser emission at $1.3 \mathrm{~cm}$ toward SVS 13 made with subarcsec angular resolution along several epochs. Our goals were to establish accurately the position of the $\mathrm{H}_{2} \mathrm{O}$ masers with respect to the components of the radio binary and to study if this relative location could shed light on the evolutionary stage of the stars. We were also interested in trying to understand from which of the stars of this binary system is the outflow originating, since high velocity $\mathrm{H}_{2} \mathrm{O}$ masers are indicators of outflow activity and can be observed with very high angular resolution. We also studied water masers in three other locations in NGC 1333.

\section{Observations}

The $\mathrm{H}_{2} \mathrm{O}$ maser observations were made with the VLA of $\mathrm{NRAO}^{1}$ in the A configuration during 1983 October 21, 1985 February 18, 1988 October 31, and 1989 February 5. The phase calibrator was $0333+321$, for which we assumed a flux density of $1 \mathrm{Jy}$ at $1.3 \mathrm{~cm}$. The observations were taken with a total bandwidth of $3.1 \mathrm{MHz}$ and 64 channels with a width of $49 \mathrm{kHz}$ each (equivalent to $0.7 \mathrm{~km} \mathrm{~s}^{-1}$ ). The data were calibrated, edited, and imaged using the software AIPS of NRAO. The synthesized beam had typical HPFW of $\sim 0$.' 1 , for maps made with natural weighting. In order to accurately compare the $\mathrm{H}_{2} \mathrm{O}$ maser positions with the $3.6 \mathrm{~cm}$ continuum positions of Anglada et al. (2000), that also used $0333+321$ as phase calibrator, we shifted the maser positions by $\Delta \alpha=+0.0047$ and

1 The National Radio Astronomy Observatory is a facility of the National Science Foundation operated under cooperative agreement by Associated Universities, Inc.

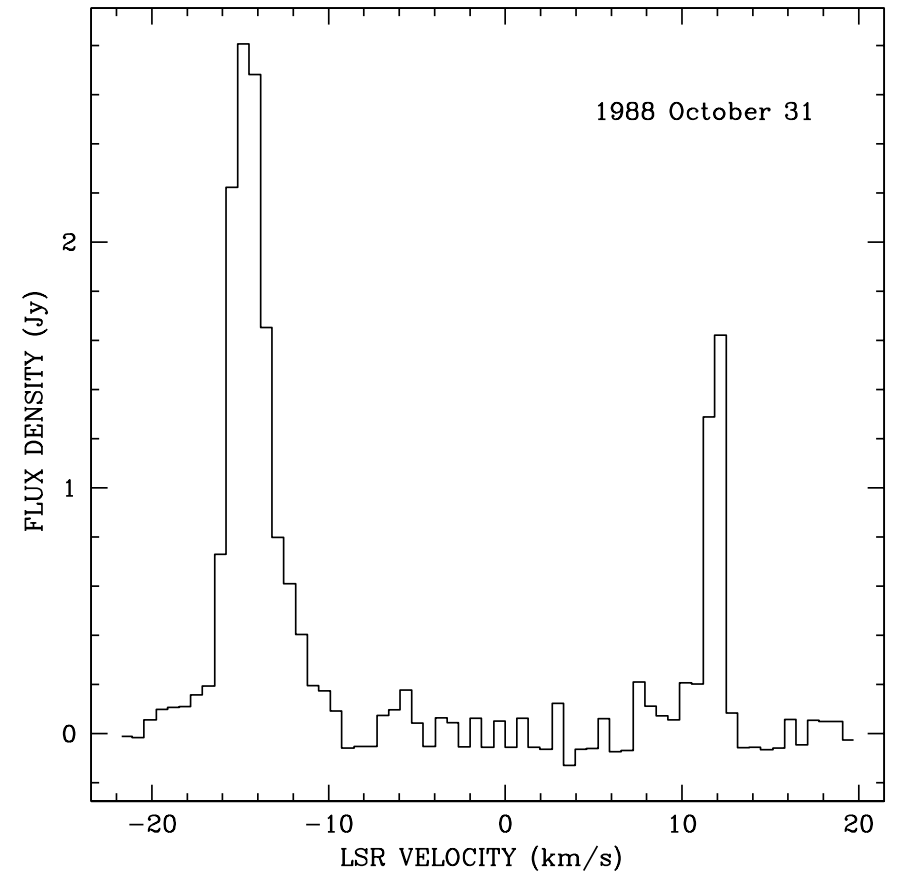

Fig. 1. $\mathrm{H}_{2} \mathrm{O}$ spectrum of SVS 13 in 1988 October 31.

$\Delta \delta=-0$.'009 to account for a recent refinement of the position of $0333+321$.

\section{Masers associated with SVS 13 (=VLA 4)}

This maser was first detected by Dickinson et al. (1974) and monitored extensively by Haschick et al. (1980), who called it $\mathrm{H}_{2} \mathrm{O}(\mathrm{A})$ to distinguish it from other masers in the region. We detected a total of eleven $\mathrm{H}_{2} \mathrm{O}$ spots associated with SVS 13 over the four epochs (one in 1983, four in 1985, four in 1988, and two in 1989). Both in 1985 and in 1988 two spots at different radial velocity (with LSR radial velocities of -20.1 and $-15.5 \mathrm{~km} \mathrm{~s}^{-1}$ for 1985 and with LSR radial velocities of -14.8 and $-5.6 \mathrm{~km} \mathrm{~s}^{-1}$ for 1988, see Fig. 1) appeared to coincide spatially within our positional uncertainty of $\sim 0$ " 02 and were considered as arising from the same position (that was taken to be the average of the two nearby positions). Furthermore, the two spots detected in 1989 coincide within 0. . 02 with spots reported in 1988 (see Table 1), and they were taken to be the same source.

We did not detect $\mathrm{H}_{2} \mathrm{O}$ emission in association with VLA 3, the source proposed by Rodríguez et al. (1997) as an alternative candidate to drive the HH 7-11 objects.

In Fig. 2 we show the maser spots overlapped on the $3.6 \mathrm{~cm}$ contour map of Anglada et al. (2000). As can be seen, the spots appear to be segregated in two velocity groups: a velocity group with $v_{\text {LSR }}$ similar to that of the ambient cloud $\left(v_{\mathrm{LSR}} \simeq 8 \mathrm{~km} \mathrm{~s}^{-1}\right)$, for which we detected five features with $v_{\mathrm{LSR}}$ in the range of 6.5 to $12.2 \mathrm{~km} \mathrm{~s}^{-1}$ and a blueshifted velocity group, for which we detected six features with $v_{\mathrm{LSR}}$ in the range of -25.4 to $-5.6 \mathrm{~km} \mathrm{~s}^{-1}$. Remarkably, all the spots in the ambient velocity group 


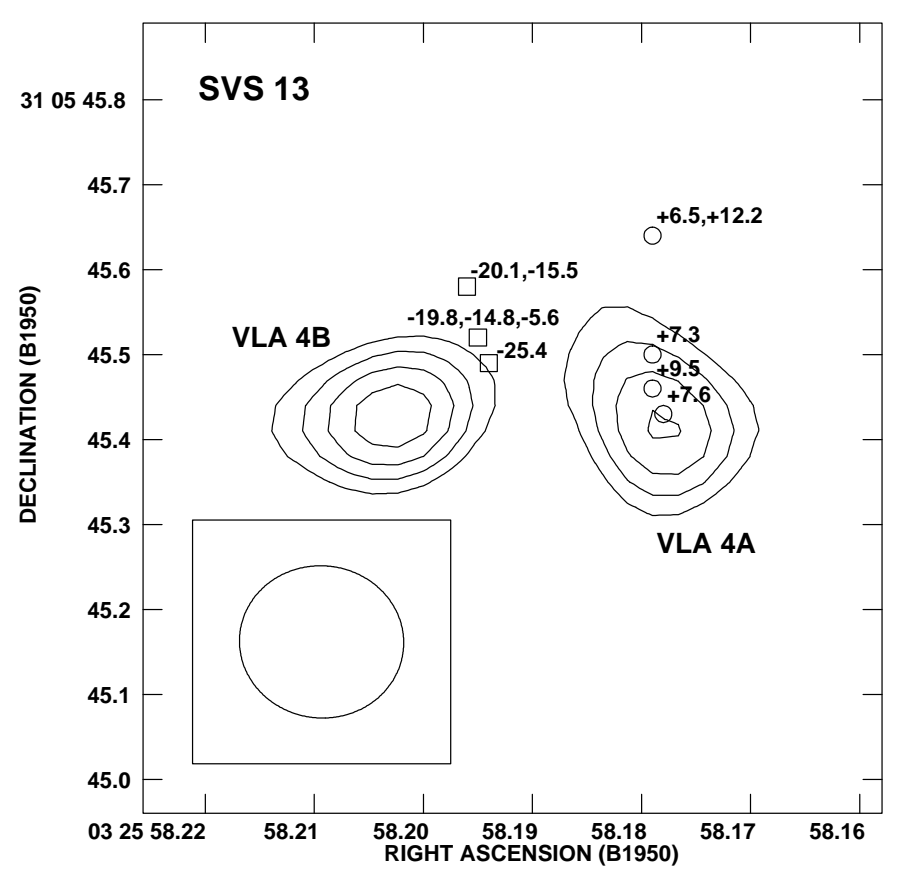

Fig. 2. Positions of the $\mathrm{H}_{2} \mathrm{O}$ spots associated with SVS 13, overlapped on the $3.6 \mathrm{~cm}$ continuum image of Anglada et al. (2000). The LSR velocity of the spots is given in the figure. The contours of the continuum emission are $-3,3,4,5$, and 6 times $14 \mu \mathrm{Jy}_{\text {beam }}{ }^{-1}$, the rms noise of the map. The half power contour of the beam of the continuum observations is shown in the bottom left corner. Note that the spots with radial velocity close to the systemic velocity $\left(v_{\mathrm{LSR}} \simeq 8 \mathrm{~km} \mathrm{~s}^{-1}\right)$, that are marked with circles, appear spatially associated with VLA 4A, while those with blueshifted radial velocity, that are marked with squares, appear spatially associated with VLA 4B.

are associated with VLA 4A, while all the spots in the blueshifted velocity group are associated with VLA 4B.

We have tried to put this result in a more quantitative manner, plotting the difference of the angular separation of the spots to the continuum sources $\mathrm{A}$ and $\mathrm{B}$, $d(\mathrm{~A})-d(\mathrm{~B})$, as a function of LSR velocity. This plot is shown in Fig. 3. It appears to us to be very significant statistically that $d(\mathrm{~A})-d(\mathrm{~B})$, in the vertical axis, is always positive for blueshifted spots (indicating that these spots are always closer to VLA4B than to VLA4A) and always negative for spots with systemic velocity (indicating that these spots are always closer to VLA4A than to VLA4B).

We believe that this result supports the suggestion of Anglada et al. (2000) that there are at least two stars, VLA 4A and VLA 4B, in association with SVS 13. If the mean radial velocity of the maser groups were indicative of the radial velocity of the stars, one could argue that VLA 4A is the more massive of the two (since its velocity is closer to the ambient cloud velocity) and that the blueshifted velocity seen in VLA 4B indicates orbital motion for this star. However, at a distance of $220 \mathrm{pc}$ (Černis 1990), the angular separation between VLA 4A and VLA $4 \mathrm{~B}$ corresponds to $\sim 65 \mathrm{AU}$, and a mass $\geq 30 M_{\odot}$ would be required for VLA 4A. The modest bolometric luminosity of the source $\left(\sim 20 L_{\odot}\right.$; Jennings et al. 1987)



Fig. 3. Difference of the angular separation of the spots to the continuum sources VLA $4 \mathrm{~A}$ and VLA $4 \mathrm{~B}, d(\mathrm{~A})-d(\mathrm{~B})$, as a function of LSR velocity. Note that $d(\mathrm{~A})-d(\mathrm{~B})$ is always positive for blueshifted spots and always negative for spots at the systemic velocity of $\sim 8 \mathrm{~km} \mathrm{~s}^{-1}$. This results corroborates the segregation of maser position as function of LSR velocity discussed in the text.

seems to rule out such a massive star. We then tentatively propose that the radial velocity of both stars is most probably close to that of the ambient cloud, and that the blueshifted velocities observed in the $\mathrm{H}_{2} \mathrm{O}$ masers associated with VLA $4 \mathrm{~B}$ are produced by outflow motions that are not present or are not as important in VLA 4A. A puzzling feature of the blueshifted masers associated with VLA $4 \mathrm{~B}$ is that they appear to the $\mathrm{NW}$ of the source, while the geometry of the large scale CO outflow (Bachiller et al. 2000) would imply that they should appear to the SE. We also note as an alternative explanation that the blueshifted masers could be part of an outflow from VLA 4A. VLBA monitoring of these masers, such as that reported by Wootten et al. (2000) should help clarify their nature.

The millimeter observations of Looney et al. (2000) do not resolve the two components of SVS 13. However, their peak position coincides, within $0{ }^{\prime \prime} 07$, with the mean position of the components VLA 4A and VLA 4B, as given by Anglada et al. (2000).

\section{Masers associated with MMS3 (=VLA 2)}

In the same field of SVS 13 (at an angular distance of $\sim 0^{\prime} .6$ to the SW) we also observed the maser called $\mathrm{H}_{2} \mathrm{O}(\mathrm{B})$ by Haschick et al. (1980). This maser was detected in 1985 , 1988, and 1989 and its parameters are given in Table 2. It is associated with a radio source reported by Haschick et al. (1980). It has a millimeter counterpart, MMS3 
Table 1. Parameters of the $\mathrm{H}_{2} \mathrm{O}$ masers associated with SVS 13 (=VLA 4).

\begin{tabular}{|c|c|c|c|c|}
\hline Epoch & $\alpha(1950)$ & $\delta(1950)$ & $\begin{array}{c}v_{\mathrm{LSR}} \\
\left(\mathrm{km} \mathrm{s}^{-1}\right)\end{array}$ & $\begin{array}{c}\text { Flux Density } \\
(\mathrm{Jy})\end{array}$ \\
\hline 1983 & 032558.179 & +310545.50 & +7.3 & 0.9 \\
\hline 1985 & 032558.194 & +310545.49 & -25.4 & 1.9 \\
\hline 1985 & 032558.196 & +310545.58 & $-20.1,-15.5$ & $0.5,1.9$ \\
\hline 1985 & 032558.179 & +310545.46 & +9.5 & 0.6 \\
\hline 1988 & 032558.195 & +310545.52 & $-14.8,-5.6$ & $2.8,0.2$ \\
\hline 1988 & 032558.178 & +310545.43 & +7.6 & 0.2 \\
\hline 1988 & 032558.179 & +310545.64 & +12.2 & 1.6 \\
\hline 1989 & 032558.194 & +310545.51 & -19.8 & 13.0 \\
\hline 1989 & 032558.178 & +310545.65 & +6.5 & 2.2 \\
\hline
\end{tabular}

Table 2. Parameters of the masers associated with MMS3 (=VLA 2).

\begin{tabular}{|c|c|c|c|c|}
\hline Epoch & $\alpha(1950)$ & $\delta(1950)$ & $\begin{array}{c}v_{\mathrm{LSR}} \\
\left(\mathrm{km} \mathrm{s}^{-1}\right)\end{array}$ & $\begin{array}{c}\text { Flux Density } \\
(\mathrm{Jy})\end{array}$ \\
\hline 1985 & 032556.406 & +310519.68 & $+8.8,+13.4$ & $0.8,1.1$ \\
\hline 1988 & 032556.409 & +310519.64 & $=+3.0,+14.1$ & $3.6,0.5$ \\
\hline 1989 & 032556.410 & +310519.65 & +1.2 & 7.5 \\
\hline 1989 & 032556.403 & +310519.62 & -2.0 & 9.7 \\
\hline
\end{tabular}

(Chini et al. 1997; Looney et al. 2000). This relatively bright radio source $(\sim 2.1 \mathrm{mJy}$ at $3.6 \mathrm{~cm})$ has been recently studied by Rodríguez et al. $(1997,1999)$, who name it VLA 2. These authors find that the radio source is a double with angular separation of $\sim 2^{\prime \prime}$ in the NS direction, and constituted by the components VLA 2(a) and VLA 2(b) (see Fig. 1 of Rodríguez et al. 1997). The water masers are, within $0 . \prime 1$, clearly associated with VLA 2(a), the brightest of the two components. This result is relevant because new observations by Reipurth et al. (2002) indicate that VLA 2(a) is the source actually associated with a star and that VLA 2(b) is most probably just a moving Herbig-Haro knot. The position of VLA 2(a) coincides within 0 .' 1 with that of its millimeter counterpart, MMS3 (SVS 13C in the nomenclature of Looney et al. 2000).

\section{Masers associated with IRAS4B (=VLA 28)}

It is unclear if this maser is the one previously identified as $\mathrm{H}_{2} \mathrm{O}(\mathrm{C})$ by Haschick et al. (1980). Its parameters are given in Table 3 . Our accurate position and that given for $\mathrm{H}_{2} \mathrm{O}(\mathrm{C})$ by Haschick et al. (1980) suggest that there is no positional coincidence and that this is a new maser. Within 0 .' 5 , all maser spots coincide spatially with the radio source VLA 28 (Mundy et al. 1993; Rodríguez et al. 1999). This source is the centimeter counterpart to the
Table 3. Parameters of the $\mathrm{H}_{2} \mathrm{O}$ masers associated with IRAS4B (=VLA 28).

\begin{tabular}{|c|c|c|c|c|}
\hline Epoch & $\alpha(1950)$ & $\delta(1950)$ & $\begin{array}{c}v_{\mathrm{LSR}} \\
\left(\mathrm{km} \mathrm{s}^{-1}\right)\end{array}$ & $\begin{array}{c}\text { Flux Density } \\
(\mathrm{Jy})\end{array}$ \\
\hline 1983 & 032606.491 & +310250.06 & -0.9 & 28.2 \\
\hline 1983 & 032606.477 & +310250.39 & +9.7 & 1.3 \\
\hline 1985 & 032606.488 & +310250.12 & -0.2 & 1.5 \\
\hline 1985 & 032606.475 & +310250.45 & +14.9 & 6.2 \\
\hline 1988 & 032606.498 & +310249.92 & +13.1 & 1.2 \\
\hline 1988 & 032606.488 & +310250.06 & +13.8 & 2.2 \\
\hline 1989 & 032606.496 & +310249.95 & +9.8 & 1.5 \\
\hline 1989 & 032606.491 & +310250.07 & +10.5 & 0.5 \\
\hline
\end{tabular}

Table 4. Parameters of the $\mathrm{H}_{2} \mathrm{O}$ masers associated with IRAS7 (=VLA 27).

\begin{tabular}{|c|c|c|c|c|}
\hline Epoch & $\alpha(1950)$ & $\delta(1950)$ & $\begin{array}{c}v_{\mathrm{LSR}} \\
\left(\mathrm{km} \mathrm{s}^{-1}\right)\end{array}$ & $\begin{array}{c}\text { Flux Density } \\
(\mathrm{Jy})\end{array}$ \\
\hline 1983 & 032605.483 & +310811.19 & +6.0 & 0.7 \\
\hline 1983 & 032605.487 & +310811.26 & +8.0 & 6.2 \\
\hline 1985 & 032605.613 & +310812.80 & +8.0 & 4.6 \\
\hline 1988 & 032605.040 & +310802.31 & +17.8 & 0.8 \\
\hline 1988 & 032605.048 & +310750.14 & +20.4 & 0.2 \\
\hline
\end{tabular}

source IRAS4B (Sandell et al. 1991) and is believed to drive a molecular outflow (Blake et al. 1995). The centimeter position of VLA 28 coincides within 0. ". 3 with that of its millimeter counterpart (Looney et al. 2000). Marvel et al. (2000) have recently reported water maser emission associated with the relatively nearby source IRAS4A. We do not detect water emission at the position of this source, which is located at $\sim 30^{\prime \prime}$ from our phase center.

\section{Masers associated with IRAS7 (=VLA 27)}

This maser was first reported by Henkel et al. (1986), who found that it had radial velocity features in the range of 4 to $15 \mathrm{~km} \mathrm{~s}^{-1}$. Our observations, made only in 1983 , 1985, and 1988, are summarized in Table 4 and show several features in the -0.9 to $+14.9 \mathrm{~km} \mathrm{~s}^{-1}$ velocity range. Henkel et al. (1986) noted that this maser is remarkable for its close association with the knots that form HH 6 . However, our positions for 1983 and 1985 show that the positional association is much closer with the radio continuum source first detected by Snell \& Bally (1986) and most recently studied by Rodríguez et al. (1999), since masers and continuum are coincident within $\sim 2^{\prime \prime}$. These last authors name this continuum source VLA 27, This source is the cm counterpart of the far-IR source IRAS7 (Jennings et al. 1987). The source is displaced $\sim 15^{\prime \prime}$ to 
the SW of the main group of knots of HH 6 (see Fig. 10 of Rodríguez et al. 1999).

The two maser spots detected in 1988 appear to be significantly redshifted in velocity with respect to the 1983 and 1985 detections. These two spots are also significantly displaced to the SW of the continuum source and may be tracing the red lobe of this outflow.

\section{Conclusions}

We presented high angular resolution VLA observations of water masers toward three regions in NGC 1333. Our main results are the following.

1. All four masers discussed here are found to be closely associated with $\mathrm{cm}$ radio continuum sources, as indicated by the comparison of our accurate positions with radio continuum positions available in the literature. The offsets between the water maser and radio continuum positions are in the range of $0 .{ }^{\prime \prime} 1-2^{\prime \prime}$.

2. The water maser $\mathrm{HH} 7 \mathrm{H}_{2} \mathrm{O}(\mathrm{A})$ is associated with SVS 13 (=VLA 4). The spots of this maser are segregated in two velocity groups: a velocity group with $v_{\text {LSR }}$ similar to that of the ambient cloud $\left(v_{\mathrm{LSR}} \simeq\right.$ $8 \mathrm{~km} \mathrm{~s}^{-1}$ ), and a blueshifted velocity group ( $v_{\mathrm{LSR}}$ in the range of -25.4 to $-5.6 \mathrm{~km} \mathrm{~s}^{-1}$ ). Remarkably, all the spots in the first velocity group are associated with VLA 4A, while all the spots in the blueshifted velocity group are associated with VLA 4B. This result supports the suggestion of Anglada et al. (2000) that there are at least two stars, VLA $4 \mathrm{~A}$ and VLA 4B, in association with SVS 13.

3. The maser $\mathrm{HH} 7 \mathrm{H}_{2} \mathrm{O}(\mathrm{B})$ is associated with VLA 2(a) (=MMS3), the brightest of the two radio components that form VLA 2.

4. Our accurate positions show that both IRAS4B (=VLA 28) and IRAS7 (=VLA 27, the exciting source of $\mathrm{HH} 6$ ) are associated with water maser emission.

5. Our data did not allow us to detect organized, systematic proper motions in the maser spots, most probably due to the short lifetime of the features. We have separations of the order of a year between our observations, while the study of Wootten et al. (2000) indicates lifetimes of only weeks for typical water masers in NGC 1333. Then, the maser spots observed at a given epoch are different to those observed at other epochs.

Acknowledgements. We thank Mark Claussen for his comments. LFR acknowledges the support from DGAPA, UNAM and CONACyT, México. GA and JMT acknowledge support from DGESIC grant PB98-0670-C02 and from Junta de
Andalucía (Spain). GA also acknowledges support from MEC, Spain, and from SAO, USA.

\section{References}

Anglada, G., Rodríguez, L. F., \& Torrelles, J. M. 2000, ApJ, 542, L123

Aspin, C., \& Sandell, G. 1994, A\&A, 288, 803

Aspin, C., Sandell, G., \& Russell, A. P. G. 1994, A\&AS, 106, 165

Bachiller, R., Guilloteau, S., Gueth, F., et al. 1998, A\&A, 339, L49

Bachiller, R., Gueth, F., Guilloteau, S., Tafalla, M., \& Dutrey, A. 2000, A\&A, 362, L33

Bally, J., Devine, D., \& Reipurth, B. 1996, ApJ, 473, 149

Blake, G. A., Sandell, G., van Dishoeck, E. F., et al. 1995, ApJ, 441,689

Černis, K. 1990, Ap\&SS, 166, 315

Chini, R., Reipurth, B., Sievers, A., et al. 1997, A\&A, 325, 542

Dickinson, D. F., Kojoian, G., \& Strom, S. E. 1974, ApJ, 194, L93

Eislöffel, J., Günther, E., Hessman, F. V., et al. 1991, ApJ, 383, L19

Goodrich, R. W. 1986, AJ, 92, 885

Grossman, E. N., Masson, C. R., Sargent, A. I., et al. 1987, ApJ, 320, 356

Haschick, A. D., Moran, J. M., Rodríguez, L. F., et al. 1980, ApJ, 237, 26

Henkel, C., Haschick, A. D., \& Güsten, R. 1986, A\&A, 165, 197

Herbig, G. H. 1974, Draft Catalog of Herbig-Haro Objects, Lick Obs. Bull. No. 658

Jennings, R. E., Cameron, D. H. M., Cudlip, W., \& Hirst, C. J. 1987, MNRAS, 226, 461

Looney, L. W., Mundy, L. G., \& Welch, W. J. 2000, ApJ, 529, 477

Marvel, K. B., Claussen, M. J., Wootten, H. A., \& Wilking, B. 2000, AAS Meeting 196, \#04.02

Mundy, L. G., McMullin, J. P., Grossman, A. W., \& Sandell, G. 1993, Icarus, 106, 11

Reipurth, B., Rodríguez, L. F., Anglada, G., \& Bally, J. 2002, AJ, submitted

Rodríguez, L. F., Anglada, G., \& Curiel, S. 1997, ApJ, 480, L125

Rodríguez, L. F., Anglada, G., \& Curiel, S. 1999, ApJS, 125, 427

Sandell, G., Aspin, C., Duncan, W. D., Russell, A. P. G., \& Robson, E. I. 1991, ApJ, 376, L17

Snell, R. L., \& Bally, J. 1986, ApJ, 303, 683

Strom, S. E., Grasdalen, G. L., \& Strom, K. M. 1974, ApJ, 191, 111

Strom, S. E., Vrba, F. J., \& Strom, K. M. 1976, AJ, 81, 314

Wootten, H. A., Marvel, K. B., Claussen, M. J., \& Wilking, B. 2000, AAS Meeting 197, \#132.05 